\title{
Gas-phase infrared spectroscopy of the rubicene cation $\left(\mathrm{C}_{26} \mathrm{H}_{14}{ }^{\bullet+}\right)$
}

\section{A case study for interstellar pentagons}

\author{
J. Bouwman ${ }^{1}$, C. Boersma ${ }^{2}$, M. Bulak ${ }^{1,3}$, J. Kamer ${ }^{1}$, P. Castellanos ${ }^{3}$, A. G. G. M. Tielens ${ }^{3}$, and H. Linnartz ${ }^{1}$ \\ ${ }^{1}$ Laboratory for Astrophysics, Leiden Observatory, PO Box 9513, 2300 RA Leiden, The Netherlands \\ e-mail: bouwman@strw.leidenuniv.nl \\ 2 NASA Ames Research Center, MS 245-6, Moffett Field, CA 94035-0001, USA \\ e-mail: Christiaan. Boersma@nasa.gov \\ ${ }^{3}$ Leiden Observatory, Leiden University, PO Box 9513, 2300 RA Leiden, The Netherlands
}

Received 28 October 2019 / Accepted 25 February 2020

\begin{abstract}
Infrared bands at 3.3, 6.2, 7.6, 7.8, 8.6, and $11.2 \mu \mathrm{m}$ have been attributed to polycyclic aromatic hydrocarbons (PAHs) and are observed toward a large number of galactic and extragalactic sources. Some interstellar PAHs possibly contain five-membered rings in their honeycomb carbon structure. The inclusion of such pentagon defects can occur during PAH formation, or as large PAHs are eroded by photo-dissociation to ultimately yield fullerenes. Pentagon formation is a process that is associated with the bowling of the PAH plane, that is, the ability to identify PAH pentagons in space holds the potential to directly link PAHs to cage and fullerene structures. It has been hypothesized that infrared (IR) activity around $1100 \mathrm{~cm}^{-1}$ may be a spectral marker for interstellar pentagons. We present an experimentally measured gas-phase IR absorption spectrum of the pentagon-containing rubicene cation $\left(\mathrm{C}_{26} \mathrm{H}_{14}{ }^{{ }^{+}}\right)$to investigate if this band is present. The NASA Ames PAH IR Spectroscopic Database is scrutinized to see whether other rubicene-like species show IR activity in this wavelength range. We find that a specific molecular characteristic is responsible for this IR band. Namely, the vibrational motion attributed to this IR activity involves pentagon-containing harbors. An attempt to find this specific mode in Spitzer observations is undertaken and tentative detections around $9.3 \mu \mathrm{m}$ are made toward the reflection nebula NGC 7023 and the H IIregion IRAS 12063-6259. Simulated emission spectra are used to derive upper limits for the contributions of rubicene-like pentagonal PAH species to the IR band at $6.2 \mu \mathrm{m}$ toward these sources.
\end{abstract}

Key words. methods: laboratory: molecular - ISM: molecules - photon-dominated region - molecular processes

\section{Introduction}

Interstellar polycyclic aromatic hydrocarbons (PAHs) have been identified by their strong and characteristic infrared (IR) emission bands at 3.3, 6.2, 7.6, 7.8, 8.6 and $11.2 \mu \mathrm{m}$ that are commonly perched on top broad emission plateaus (Leger \& Puget 1984; Allamandola et al. 1985). Aromatic IR bands have been detected toward a large number of interstellar sources and are used as tracers of the local physical conditions (e.g., Tielens 2008). It is generally accepted that the aromatic IR bands are emitted by PAHs that relax to the ground state after being excited by a single interstellar UV photon (Allamandola et al. 1989). Interstellar PAHs are believed to be large, containing more than 50 carbon atoms, and exist in various charge states (e.g., Joblin et al. 1996; Galliano et al. 2008). It has been estimated that about $15 \%$ of the total cosmic carbon budget is locked up in PAHs, suggesting that they play a crucial role in the cosmochemical cycle of organic matter (Tielens 2008).

A large variety of mechanisms are expected to contribute to the formation of PAHs in the combustion of fossil fuels (Richter \& Howard 2000; Moriarty \& Frenklach 2000; Parker et al. 2014; Kaiser et al. 2015; Yang et al. 2017), some of which are applied to astrochemistry to explain the formation of interstellar aromatic molecules in the outflows of carbon-rich stars (e.g., Frenklach \& Feigelson 1989; Cherchneff et al. 1992). The sequences of chemical reactions involved not only produce clean hexagon containing aromatic molecules, but they have also been shown in the laboratory to result in pentagon containing species (Bouwman et al. 2015; Johansson et al. 2018; Commodo et al. 2019; Schulz et al. 2019; McCabe et al. 2020). Pentagon inclusion is also invoked to explain the formation of fullerenes through erosion of large PAHs as this provides a way to bowl the planar PAH plane (Berné \& Tielens 2012). Laboratory spectroscopic evidence for pentagon formation upon PAH photodissociation has indeed been reported for PAH species containing up to three aromatic rings (Bouwman et al. 2016; de Haas et al. 2017). Pentagon-bearing polyaromatic species are thus expected to be present in the interstellar medium (ISM).

A vast amount of literature is available on the IR spectroscopy of cationic interstellar relevant PAHs, employing both matrix isolation spectroscopy as well as gas-phase ion trap action spectroscopy (e.g., Hudgins et al. 1994; Hudgins \& Allamandola 1995a,b, 1997; Oomens et al. 2003, 2006; Zhen et al. 2017; Bouwman et al. 2019). Most of these experiments have focused on PAH radical cations consisting of hexagonal carbon homocycles and only a few measurements have been reported on aromatic molecules that contain a single pentagonal ring. It was noted by Ekern et al. (1998) that some pentagon-containing PAHs exhibit enhanced photostability toward the break-down of their carbon skeleton, but they mentioned that this has yet to be verified for larger species. Hudgins et al. (2000) reported a matrix isolation spectroscopic study of seven PAHs containing a cyclopentadienyl ring and their corresponding ions. Oomens et al. (2001) 


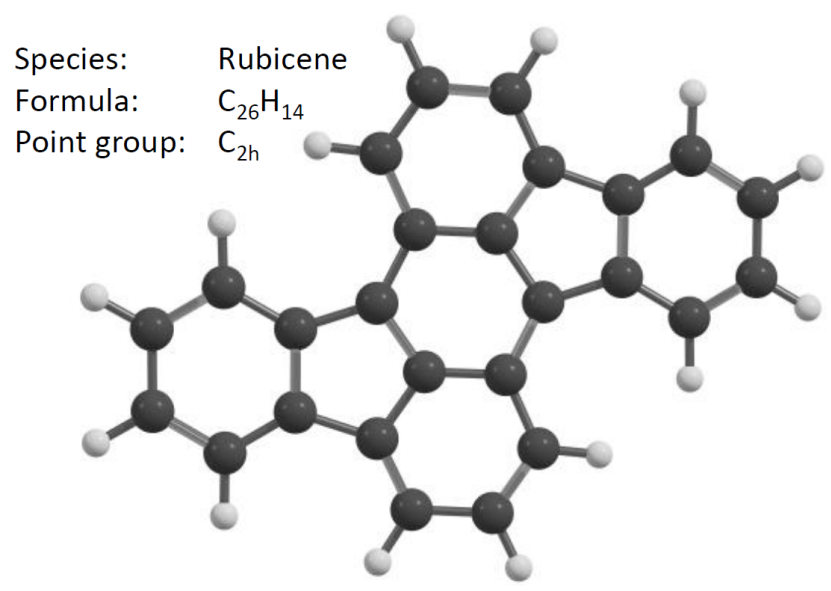

Fig. 1. Molecular structure of rubicene shown together with its formula and point group.

measured gas-phase spectra of a few small pentagon-containing PAHs, including fluoranthene, which has aromatic character over the pentagon unit. Later, Galué et al. (2011) measured the IR spectrum of the coranulene radical cation and noted enhanced intensity around $1100 \mathrm{~cm}^{-1}$ with respect to the spectrum of the (nonpentagon containing) coronene radical cation. Most recently, Zhen et al. (2018) have characterized the IR fingerprint spectrum of the diindenoperylene cation $\left(\mathrm{C}_{32} \mathrm{H}_{16}{ }^{\bullet+}\right)$ that contains two pentagons, and they suggest that vibrational modes between 1000 and $\sim 1150 \mathrm{~cm}^{-1}$ possibly mark the presence of the pentagon ring structures. Early studies on the influence of pentagons on the spectra of interstellar PAHs focused on the $500-700 \mathrm{~cm}^{-1}$ range (Moutou et al. 2000; Van Kerckhoven et al. 2000; Boersma et al. 2010).

In this work, we extend on previous findings by investigating the mid-IR spectrum of a polyaromatic molecule containing two isolated pentagons that participate in the aromatic structure of the molecule. We chose to measure the spectrum of the rubicene radical cation $\left(\mathrm{Rubi}^{{ }^{++}}\right.$; Fig. 1), as it contains pentagons and is a fully aromatic system like buckminster fullerene. We show, in agreement with earlier studies on pentagon containing aromatics, that a band appears at $1100 \mathrm{~cm}^{-1}$, which is representative for vibrations caused by pentagonal defects in the otherwise hexagonal aromatic structures. We scrutinize the NASA Ames PAH IR Spectroscopic Database (Bauschlicher et al. 2018; Boersma et al. 2014, Mattioda et al., in prep.) to investigate the potential bearing of pentagon-containing PAHs like Rubi ${ }^{\bullet+}$ on the astronomical IR emission spectrum. Tentative detections of the pentagon IR feature between $1100-1200 \mathrm{~cm}^{-1}(\sim 9.3 \mu \mathrm{m})$ are made in lowresolution Spitzer (Werner et al. 2004) spectra obtained using the IR spectrograph (IRS; Houck et al. 2004).

The outline of the paper is as follows. Section 2 sets out the experimental and computational methods, followed by Sect. 3 where the obtained results are presented. Next, Sect. 4 discusses the results and puts forward the astronomical implications. Section 5 provides a summary and conclusions.

\section{Methods}

\subsection{Experimental setup}

The mid-IR spectrum of the rubicene radical cation was recorded on the instrument for photodissociation of PAHs (i-PoP) connected to the free electron laser for IR experiments (FELIX;
Oepts et al. 1995). A complete description of the apparatus, i-PoP, is available from a previous publication (Zhen et al. 2014), and here we only provide a concise description of the relevant experimental details.

A quadrupole ion-trap (QIT) is mounted inside a vacuum chamber pumped down to a base pressure of $\sim 10^{-8}$ mbar. An oven containing the rubicene sample (Rubi; $\mathrm{C}_{26} \mathrm{H}_{14}$; Kentax; $\geq 99.5 \%$; Fig. 1) is also mounted inside this vacuum chamber and is situated just below an electron gun. Rubicene molecules were gently sublimated by heating the oven to a temperature of $420 \mathrm{~K}$. Once in the gas phase, the molecules were ionized by electron ionization (EI) at an energy of $85 \mathrm{eV}$. Ions were steered into the QIT using electrostatic lenses and were trapped in the $1 \mathrm{MHz}$, $2100 \mathrm{~V}_{\mathrm{t}-\mathrm{t}}$ electric field of the trap. Ions were accumulated inside the QIT during $1.2 \mathrm{~s}$ for each trap fill in the duty cycle. An electrostatic gate was closed when the trap was filled and ions were mass selected by applying a Stored Waveform Inverse Fourier Transform (SWIFT) pulse to the repeller endcap of the ion trap according to the method by Doroshenko \& Cotter (1996). Next, the mass-selected ions were exposed to the IR radiation from the free electron laser. A high-voltage pulse of $-800 \mathrm{~V}$ and $+800 \mathrm{~V}$ was subsequently applied to the extractor and repeller endcaps, respectively, to eject the (fragment) ions into a reflectron type mass spectrometer. The time-of-flight (TOF) mass spectrum of the ions was recorded by measuring the flight time to the Z-gap type microchannel plate (MCP) detector. Helium was admitted to the trap assembly, resulting in a constant pressure of $\sim 1 \times 10^{-6}$ mbar. This helped to cool the ion cloud and reduced its size, and improved the mass resolution, which amounts to $m / \Delta m \approx 900$ for the experiments reported here.

IR spectra were recorded over the $600-1700 \mathrm{~cm}^{-1}$ range by means of IR multiple-photon dissociation (IRMPD) spectroscopy using the radiation of FELIX. The light entered the vacuum chamber through a $\mathrm{KBr}$ window and was tightly focused onto the ion cloud in the center of the trap. A mechanical shutter was placed just in front of the entrance window to ensure that the ion cloud was exposed to a single free electron laser (FEL) pulse after the trap fill and SWIFT filtering had completed. The shutter was placed in a box that was purged with dry nitrogen to prevent a loss of IR laser power through atmospheric absorption. The free electron laser was operated at $10 \mathrm{~Hz}$ and had a maximum pulse energy of $120 \mathrm{~mJ}$ at a macropulse duration of $6 \mu \mathrm{s}$. About $60 \mathrm{~mJ}$ of the energy was effectively coupled into the ion trap.

IRMPD spectra were obtained by recording the wavelength dependent dissociation mass spectrum of the trapped Rubi ${ }^{\bullet+}$. A Pearson IV function was fitted to each of the mass peaks observed in every recorded mass spectrum to determine the integrated intensity of the signal. The dissociation yield was then calculated by dividing the sum of the integrated intensity of all fragment peaks by the total integrated ion intensity (i.e., fragment plus parent). The laser was tuned from 600 to $1700 \mathrm{~cm}^{-1}$ in steps of $3 \mathrm{~cm}^{-1}$ and an average of three mass spectra was recorded at each photon energy to enhance the signal-to-noise ratio $(\mathrm{S} / \mathrm{N})$. Saturation occurs when all of the parent ions in the trap are dissociated using a single FEL macropulse, resulting in flat top spectral features in the IRMPD spectrum. Spectra have been recorded using the full laser power in the trap (60 $\mathrm{mJ}$ macropulse energy) as well as at $5 \mathrm{~dB}$ attenuation (resulting in a macropulse energy of $20 \mathrm{~mJ}$ ) to prevent saturation of the strongest bands between 1200 and $1700 \mathrm{~cm}^{-1}$. A power scan was performed before and after each experiment, allowing for a firstorder correction of the dissociation yield to power. We note that IRMPD signal strengths exhibit a nonlinear intensity response. 


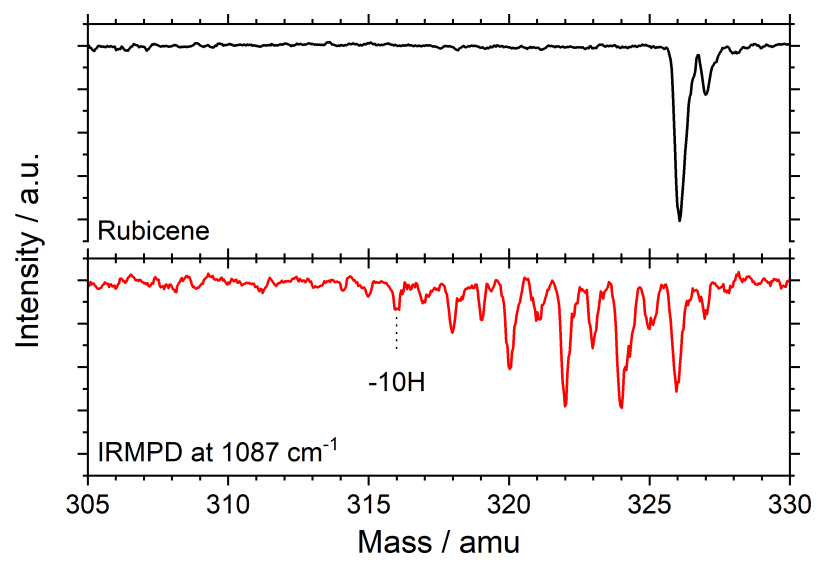

Fig. 2. Typical mass spectra of isolated Rubi ${ }^{\bullet+}$ (top) and the rubicene cation after being resonantly excited at $1087 \mathrm{~cm}^{-1}$ (bottom). The intensity of the lower panel is multiplied by a factor of three for presentation purposes.

\subsection{Computational method}

The structure of rubicene was optimized at the B3LYP/6$311++\mathrm{G}(\mathrm{d}, \mathrm{p})$ level of theory using the Gaussian09 suite of programs (Frisch et al. 2009). The resulting harmonic frequencies were scaled using a uniform scaling factor of 0.965 (Andersson \& Uvdal 2005) to account for anharmonicity effects. The scaled vibrational normal modes were convolved with a Gaussian function with a $30 \mathrm{~cm}^{-1}$ full-width-at-half-maximum (FWHM) to allow for a comparison with the laboratory measured IR spectra.

\section{Results}

In this section we discuss the experimental mass spectrometric and spectroscopic results for the prototypical pentagon-bearing molecule Rubi ${ }^{\bullet+}$. The basic strategy is to identify the spectral feature(s) typical for a pentagon structure in a polycyclic aromatic geometry that could be searched for in astronomical spectra.

\subsection{Rubicene mass spectrometry}

The top panel of Fig. 2 shows a mass spectrum of Rubi ${ }^{\bullet+}$ after being SWIFT isolated, and prior to being exposed to IR radiation. Peaks are visible that correspond to the Rubi ${ }^{\bullet+}$ parent peak at $m / z 326$ and the ${ }^{13} \mathrm{C}$ isotopologues at $\mathrm{m} / z 327$ and 328. The bottom panel of Fig. 2 displays a mass spectrum that is recorded after the trapped $\mathrm{Rubi}^{\bullet+}$ has been exposed to radiation from FELIX, operated without attenuation of power and tuned to a frequency of $1087 \mathrm{~cm}^{-1}$ (a pentagon-related mode, vide infra) to induce IRMPD. The impacting radiation causes the Rubi ${ }^{\bullet+}$ to sequentially lose $\mathrm{H}$-atoms, resulting in a pattern showing more intense peaks for the loss of an even number of H-atoms compared to those corresponding to the loss of an odd number of H-atoms (cf. Castellanos et al. 2018). A train of peaks corresponding to the loss of up to ten $\mathrm{H}$-atoms from the parent ion is clearly visible. Also, the parent ion is still visible in the mass spectrum, indicating that the IR absorption band is not saturated.

\subsection{Rubicene spectroscopy}

Mass spectra such as those presented in Fig. 2 were recorded between 600 and $1700 \mathrm{~cm}^{-1}$ in steps of $3 \mathrm{~cm}^{-1}$. The IRMPD induced peaks in the mass spectra are fitted and integrated, and

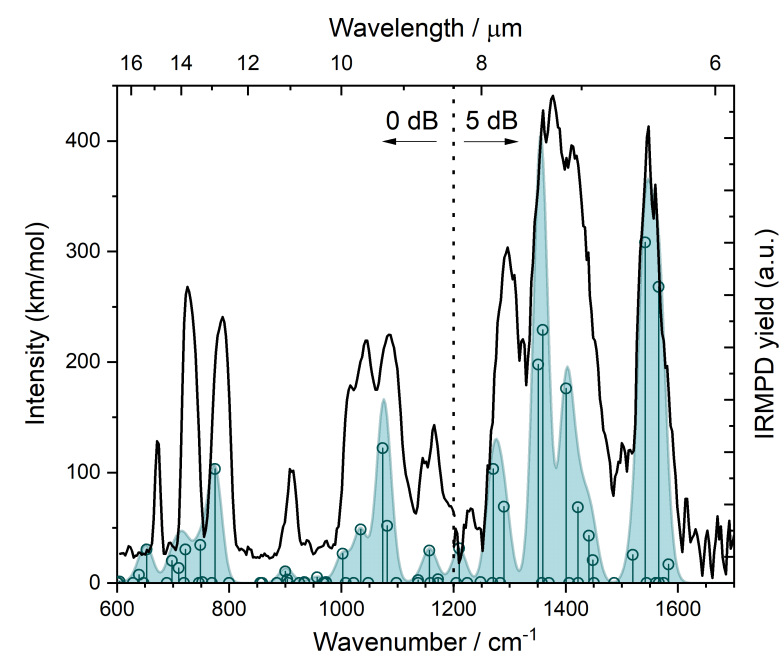

Fig. 3. Spectrum of Rubi ${ }^{\bullet+}$ recorded over the $600-1700 \mathrm{~cm}^{-1}$ range shown together with the computed stick spectrum as well as the computed spectrum convolved with a $30 \mathrm{~cm}^{-1}$ broad Gaussian line profile (turquoise). The $1200-1700 \mathrm{~cm}^{-1}$ range has been recorded at an IR laser attenuation of $5 \mathrm{~dB}$ to avoid saturation.

dissociation yields are subsequently determined. The dissociation yield is plotted as a function of frequency in Fig. 3, resulting in a fingerprint IR spectrum of Rubi ${ }^{\bullet+}$. This spectrum is composed of two parts. The first part between 600 and $1200 \mathrm{~cm}^{-1}$ is recorded at $0 \mathrm{~dB}$ attenuation of the laser power, that is, exposing the ion cloud to the full macropulse energy emitted by FELIX. The part of the spectrum between 1200 and $1700 \mathrm{~cm}^{-1}$ is recorded at $5 \mathrm{~dB}$ attenuation to avoid saturation of bands, that is, preventing full dissociation of the parent ion. A first-order correction of IRMPD yields to FEL power is applied, resulting in the combined spectrum shown in Fig. 3.

The computed IR spectrum of $\mathrm{Rubi}^{\bullet+}$, represented by turquoise circles and drop lines, is also shown in Fig. 3. The frequencies of the computed normal modes were scaled by a factor of 0.965 to account for anharmonicity effects (Andersson \& Uvdal 2005). Also shown, in turquoise in this figure, is the result of a convolution of the stick spectrum with a Gaussian line profile with a FWHM of $30 \mathrm{~cm}^{-1}$. The measured and computed spectra are slightly offset in intensity with respect to each other to facilitate comparison of absorption bands. Overall, the computed band positions fall within $20 \mathrm{~cm}^{-1}$ of the measured band positions. There is a mismatch, however, between the relative intensities of the measured IRMPD spectrum and the computed and convolved spectrum. This is commonly observed in a comparison of IRMPD spectra with DFT computed spectra and is caused by the nonlinear nature of the IRMPD process (Oomens et al. 2000), which is difficult to correct for. This effect is particularly strong for the spectrum shown in Fig. 3, as the lowand high-frequency parts have been recorded at two different laser power settings. Saturation is prevented in the high-frequency side of the spectrum, but the nonlinear effect is very pronounced.

The measured IR absorption bands were fitted using multicomponent Gaussians to determine centroids for the band to compare them with the scaled computed normal mode frequencies. The spectrum is fitted very well with 16 individual Gaussian line profiles (see Fig. A.1). The centroids of the Gaussians are listed in Table 1, together with band intensities and symmetry of the DFT computed modes. Only modes with computed IR intensities exceeding $20 \mathrm{~km} \mathrm{~mol}^{-1}$ are listed in this table. From a comparison of the bands in Table 1 it can be seen that, overall, a good match exists between the computed bands 
Table 1. Comparison between the measured band positions and the calculated normal modes scaled by a factor of 0.965 to account for anharmonicitiy effects.

\begin{tabular}{lccc}
\hline \hline Measured & \multicolumn{3}{c}{ Calculated } \\
\hline$v_{k}$ & $\begin{array}{c}v_{k} \\
\mathrm{~cm}^{-1}\end{array}$ & $\begin{array}{c}\sigma \\
\mathrm{km} \mathrm{mol}^{-1}\end{array}$ & Symmetry \\
\hline 672 & 653 & 30 & $\mathrm{~A}_{u}$ \\
$\ldots$ & 698 & 20 & $\mathrm{~A}_{u}$ \\
730 & 722 & 30 & $\mathrm{~A}_{u}$ \\
$\ldots$ & 749 & 34 & $\mathrm{~A}_{u}$ \\
786 & 775 & 103 & $\mathrm{~A}_{u}$ \\
911 & $-(a)$ & $-(a)$ & $-(a)$ \\
1012 & 1002 & 27 & $\mathrm{~B}_{u}$ \\
1041 & 1034 & 49 & $\mathrm{~B}_{u}$ \\
1087 & 1074 & 122 & $\mathrm{~B}_{u}$ \\
$\ldots$ & 1082 & 52 & $\mathrm{~B}_{u}$ \\
1163 & 1157 & 29 & $\mathrm{~B}_{u}$ \\
1229 & 1210 & 31 & $\mathrm{~B}_{u}$ \\
1294 & 1271 & 103 & $\mathrm{~B}_{u}$ \\
$\ldots$ & 1290 & 69 & $\mathrm{~B}_{u}$ \\
1364 & 1351 & 198 & $\mathrm{~B}_{u}$ \\
$\ldots$ & 1359 & 229 & $\mathrm{~B}_{u}$ \\
1419 & 1401 & 176 & $\mathrm{~B}_{u}$ \\
$\ldots$ & 1422 & 69 & $\mathrm{~B}_{u}$ \\
1466 & 1441 & 43 & $\mathrm{~B}_{u}$ \\
$\ldots$ & 1449 & 21 & $\mathrm{~B}_{u}$ \\
1502 & 1519 & 26 & $\mathrm{~B}_{u}$ \\
1543 & 1541 & 308 & $\mathrm{~B}_{u}$ \\
1571 & 1566 & 268 & $\mathrm{~B}_{u}$ \\
\hline & & & \\
\hline
\end{tabular}

Notes. Also shown are the intensity and symmetry of the computed bands. Only transitions with IR intensities exceeding $20 \mathrm{~km} \mathrm{~mol}^{-1}$ are listed. (a) The measured band overlaps with frequencies of a number of computed bands that are close in energy but have intensities $\leq 20 \mathrm{~km} \mathrm{~mol}^{-1}$.

and the experimentally determined band centroids, although some computed modes overlap in the experimental data. The biggest discrepancy is seen in the band measured at $911 \mathrm{~cm}^{-1}$ that has no explicit computed counterpart listed in the table. This measured band is likely caused by a number of weak modes $\left(<20 \mathrm{~km} \mathrm{~mol}^{-1}\right)$ that spectrally overlap.

Inspection of the molecular motions involved in the various vibrational normal modes of $\mathrm{Rubi}^{\bullet+}$ indicates that the two pentagons play a pivotal role for the band measured at $1087 \mathrm{~cm}^{-1}$ (see Fig. 4). The detection of an IR band in this particular wavelength region is in line with an earlier IR spectroscopic study on the pentagon-containing species diindenoperylene (Zhen et al. 2018). We turn to the NASA Ames PAH IR Spectroscopic Database (PAHdb hereafter; Bauschlicher et al. 2018; Boersma et al. 2014, Mattioda et al., in prep.) to put the result on $\mathrm{Rubi}^{\bullet+}$ in the broader context of pentagon containing PAHs.

\section{Discussion}

We first make an inventory of pentagon-containing species in PAHdb, followed by a discussion of their general IR spectroscopic signatures. Then, we focus on the $1100 \mathrm{~cm}^{-1}$ $(\sim 9.3 \mu \mathrm{m})$ band as a possible tracer for pentagons, identify the molecular vibrations involved, and make a tentative connection with astronomical observations before drawing possible astronomical implications.

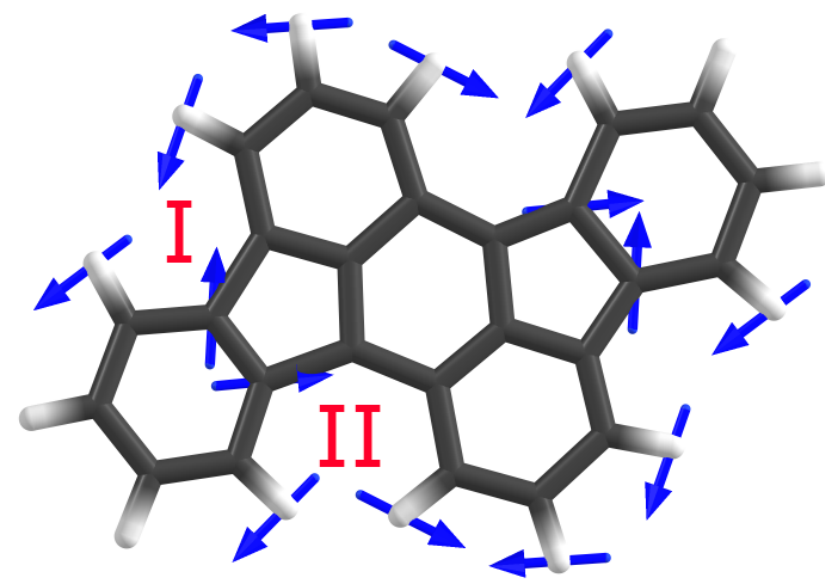

Fig. 4. Visualization of Rubi ${ }^{\bullet+}$ 's vibrational normal mode (blue vectors) computed at $1074 \mathrm{~cm}^{-1}$ (scaled value), which corresponds to the $1087 \mathrm{~cm}^{-1}$ mode in the IRMPD spectrum. The two types of "harbors" are indicated with red roman numerals. See Sects. 4.2 and 4.3 for details.

\subsection{Pentagons-bearing species in PAHdb}

The library of computed spectra at version 3.00 from PAHdb contains the spectra of 651 species with at least one pentagon. Of these, 591 are fully dehydrogenated. In order to maintain a connection with Rubi $^{\bullet+}$, we limit ourselves to those 60 pentagoncontaining PAHs that are decorated with hydrogen (see Fig. A.2 for their molecular structure). It is important to realize that 19 of these also have one or more other deformations (e.g., a 7-membered ring) and 11 have extra hydrogens. The 60 pentagon-containing species initially under consideration probe 27 distinct conformations and break down into 12 anions, 25 neutrals, and 23 cations.

\subsection{Spectral characteristics of pentagon containing PAHs}

The average $600-1700 \mathrm{~cm}^{-1}$ zero-Kelvin predicted absorption spectrum of the 60 pentagon containing PAHs is shown in the top panel of Fig. 5. Since astronomical observations are in emission, the middle panel of the figure shows the average emission spectrum, modeled with each PAH absorbing $7 \mathrm{eV}$ in energy, following the entire cooling cascade and red-shifting band positions by a fixed $15 \mathrm{~cm}^{-1}$ to mimic anharmonic effects (see e.g., Boersma et al. 2013). In reality, the anharmonic shift varies with frequency. Analysis of the anharmonic behavior of a large number of PAHs reveals that the calculated anharmonic band positions show a narrow trend with requency, independent of the detailed molecular structure (Mackie et al., in prep.). Joblin et al. (1995) measured the anharmonic shift of the strongest PAH bands for a number of species as a function of temperature. Using their results, we also model the emission spectrum of each PAH with bands having a temperature-dependent red shift. Here, first the maximum attained temperature for a species is calculated after it has absorbed a $7 \mathrm{eV}$ photon. Next, the anharmonic shift of the strongest bands of coronene at this temperature are determined from the relationships found by Joblin et al. (1995). Red shift are then interpolated from these. The resulting simulated average emission spectrum is shown in the bottom panel of Fig. 5. The two emission spectra show similar but not identical patterns. The small differences reflect that smaller molecules reach a higher temperature after absorbing the same energy and hence, their bands experience a larger red shift than larger species. 
In all three spectra the bands have been convolved with a Gaussian line profile with a FWHM of $30 \mathrm{~cm}^{-1}$. Each spectrum shows a broad plateau between $\sim 1000-1700 \mathrm{~cm}^{-1}$ with distinctive peaks on top that coincide with the astronomical UIR bands at $6.2,7.6,7.8,8.6$ and $11.2 \mu \mathrm{m}$. The average spectra for the anions, neutrals and cations alone are also shown. It is noted that there is considerable spread - signified by the $1 \sigma$ gray envelopes in Fig. 5 - for the average absorption spectrum, but comparably less for those in emission. The spectrum with the temperaturedependent shift shows blending of bands that are separated in the spectrum with the fixed shift. Notable in this regards is the band near $8.6 \mu \mathrm{m}$, which seemingly merges with that around $9.3 \mu \mathrm{m}$. Clearly, anharmonicity can have a profound effect on the PAH emission bands and, while the simple model used here provides some insight, a complete treatment of anharmonicity is far more involved and well beyond the scope of this paper (see e.g., Mackie et al. 2018).

\subsection{The $1100 \mathrm{~cm}^{-1}$ mode}

In this work we show, in agreement with the study by Zhen et al. (2018) on diindenoperylene, that pentagon containing PAHs have a distinct feature around $1100 \mathrm{~cm}^{-1}$. Indeed, the emission spectrum in Fig. 5 also shows such a feature around $1100 \mathrm{~cm}^{-1}$ (cf. Fig. 3). Perusal of the individual vibrational modes around this frequency in the different species considered here shows that this mode in $\mathrm{Rubi}^{\bullet+}$ - and diindenoperylene-like species can be attributed to a distinctive structural characteristic. Looking at this structural feature in Rubi ${ }^{\bullet+}$ (Fig. 4) itself shows the role of the four large bay-like regions, where the protruding hydrogen atoms are situated like breakwaters protecting a harbor. The visualization of the strongest mode for $\mathrm{Rubi}^{\bullet+}$, calculated at $1074 \mathrm{~cm}^{-1}$ (cf. Table 1), in Fig. 4 reveals the breakwater hydrogens performing an in-plane bending motion. These "harbors" come in two flavors with the five-membered ring playing a pivotal role in both. In the first flavor (Type I) there are three harbor walls, while in the second (Type II) there are four. Rubi ${ }^{\bullet+}$ is a particularly interesting species in this regard, as it contains two harbors of each flavor, whereas diindenoperylene has only Type I harbors. Table 2 lists the five species, in several charge states, in our PAHdb sample having this so-called harbor mode. The table shows that this mode consistently falls around $1020-1140 \mathrm{~cm}^{-1}$ and is consistently stronger in the cations.

Pentagon-containing species like Rubi ${ }^{\bullet+}$ also show the bands that coincide with the astronomical UIR emission band positions. These can be used to derive a rough estimate for the strength of a potential $1100 \mathrm{~cm}^{-1}(\sim 9.3 \mu \mathrm{m})$ feature in astronomical observations. To that end, we synthesize emission spectra, per Sect. 4.2, for the PAH cations in Table 2 and add them together (see Fig. A.3). We focus on the $6.2 \mu \mathrm{m}$ feature, which is commonly associated with PAH cations, and note that the synthesized spectrum has no distinct feature at $8.6 \mu \mathrm{m}$. The $6.2 \mu \mathrm{m}$ bands is isolated by subtracting straight-line continuum and integrated, yielding a 9.3/6.2 $\mu \mathrm{m}$ band strength ratio of $0.16 \pm 0.019$.

\subsection{Search for interstellar pentagon signatures}

The mid-IR spectra of a number of astronomical objects of different types are inspected for the presence of a distinct feature between $\sim 1028-1094 \mathrm{~cm}^{-1}(9.14-9.7 \mu \mathrm{m}$; per Table 2). Our sample is made up of the five representative low-resolution Spitzer-IRS spectra from Boersma et al. (2010), which consists of observations of a Herbig Ae/Be star (HD 36917); a planetary

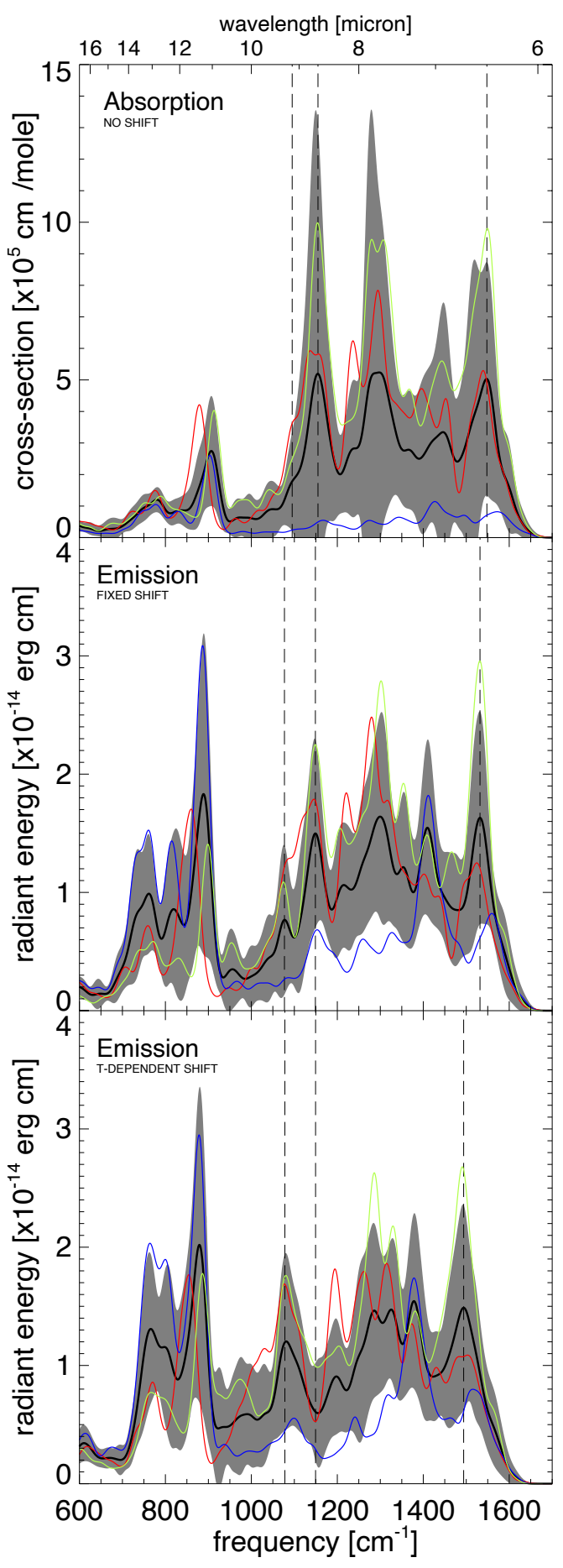

Fig. 5. Black lines show the average $600-1700 \mathrm{~cm}^{-1}$ zero-Kelvin absorption (top panel) and emission spectra (middle and bottom pan$e l s)$ of the sixty selected pentagon-containing species in PAHdb. The bands have been convolved with a Gaussian line profile with a FWHM of $30 \mathrm{~cm}^{-1}$. In the middle panel, a fixed red shift of $15 \mathrm{~cm}^{-1}$ is used for each band, while in the bottom panel the shifts depend on frequency of the mode and maximum attained temperature. The $1 \sigma$ variation around the mean is indicated by the gray shaded area and the average spectra for the anion, neutral and cation species alone are shown in red, blue, and green, respectively. Long-dashed lines are centered on the peaks of the features associated with bands at 9.3, 8.6 and $6.2 \mu \mathrm{m}$. See Sect. 4.2 for details.

nebula (NGC 6567); an H II-region (IRAS 12063-6259); a reflection nebula (NGC 7023) and a SINGS (Kennicutt et al. 2003) 
Table 2. Positions of the harbor modes in PAH species besides Rubi ${ }^{\bullet+}$ retrieved from the PAHdb.

\begin{tabular}{ccccc}
\hline \hline UID & $\begin{array}{c}v_{k}(a) \\
\mathrm{cm}^{-1}\end{array}$ & $\begin{array}{c}\sigma_{\text {int }} \\
\mathrm{km} \mathrm{mol}^{-1}\end{array}$ & Charge & Layout \\
\hline \multicolumn{5}{c}{$(b)$} \\
\hline 387 & 1139 & 8 & 0 & Type I \\
388 & 1094 & 97 & +1 & Type I \\
\hline \multicolumn{5}{c}{$\mathrm{H}_{10}$} \\
\hline 391 & 1048 & 0.6 & 0 & Type I \\
392 & 1034 & 199 & +1 & Type I \\
\hline \multicolumn{5}{c}{$\mathrm{H}_{12}$} \\
\hline 146 & 1023 & 11 & 0 & Type I \\
147 & 1028 & 23 & +1 & Type I \\
\hline \multicolumn{5}{c}{$\mathrm{C}_{20}$} \\
\hline 390 & 1048 & $\mathrm{C}_{20} \mathrm{H}_{12}$ & & \\
391 & 10099 & 47 & 0 & Type II \\
397 & 1097 & 37 & -1 & Type II \\
\hline \multicolumn{5}{c}{} \\
\hline 395 & 1125 & $\mathrm{C}_{20} \mathrm{H}_{12}$ & & Type I/II \\
396 & 1087 & 35 & 0 & Type I/II \\
398 & 1098 & 0.3 & -1 & Type I/II \\
\hline
\end{tabular}

Notes. See Sect. 4.3 for details. ${ }^{(a)}$ Unique identifiers used by PAHdb. (b) Layout of the species" "harbors".

galaxy (average spectrum from Smith et al. 2007). The reader is referred to Boersma et al. (2010) for further details. Figure 6 presents the continuum subtracted $5.2-10 \mu \mathrm{m}$ spectra and indicates the positions of the major PAH bands, molecular hydrogen lines, and where roughly the $9.3 \mu \mathrm{m}$ feature from rubicene-like pentagon-containing PAHs could be expected.

Each panel of the figure shows the typical characteristics of an astronomical PAH emission spectrum. That is, strong emission features at $6.2,7.6,7.8$ and $8.6 \mu \mathrm{m}$ perched on top of a broad plateau. Focusing on the pentagon region, it immediately becomes clear that the contribution of rubicene-like pentagoncontaining PAHs to the spectra, if any, is very weak. Nonetheless, zooming in on this region does tentatively show a feature in the spectra for three out of the five objects considered here; NGC 7023, HD 36917 and IRAS 12063-6259. However, we do note that the spectrum of HD 36917 is particular complex with a strong silicate signature (see e.g., Boersma et al. 2008, 2010), which we attempted to correct for by using a spline (see Boersma et al. 2010). Obviously, this simple approach is inadequate to capture the intricacies of silicate emission (see e.g., Juhász et al. 2010) and explains why the PAH plateau of HD 36917's spectrum in Fig. 6 differs from the others'.

The 3-tuples made up of the integrated flux (in $10^{-22} \mathrm{~W} \mathrm{~cm}^{-2}$ ), centroid (in $\mu \mathrm{m}$ ) and FWHM (in $\mu \mathrm{m}$ ) are (3.3, 9.33 $\pm 0.003,0.093$ $\pm 0.013)$ and $(1.4,9.37 \pm 0.015,0.061 \pm 0.049)$ for NGC 7023 and IRAS 12063-6259, respectively. Taking the 9.3/6.2-ratio from Sect. 4.3 , some $1.3 \pm 0.16 \%$ and $3.1 \pm 0.37 \%$ of the $6.2 \mu \mathrm{m}$ band intensity can be attributed to $\mathrm{PAH}$ cations containing pentagons and their associated harbors in the spectrum of NGC 7023 and IRAS 12063-6259, respectively.

\subsection{Astronomical implications}

The detection of fullerenes in space (Cami et al. 2010; Sellgren et al. 2010; Boersma et al. 2010) unequivocally points

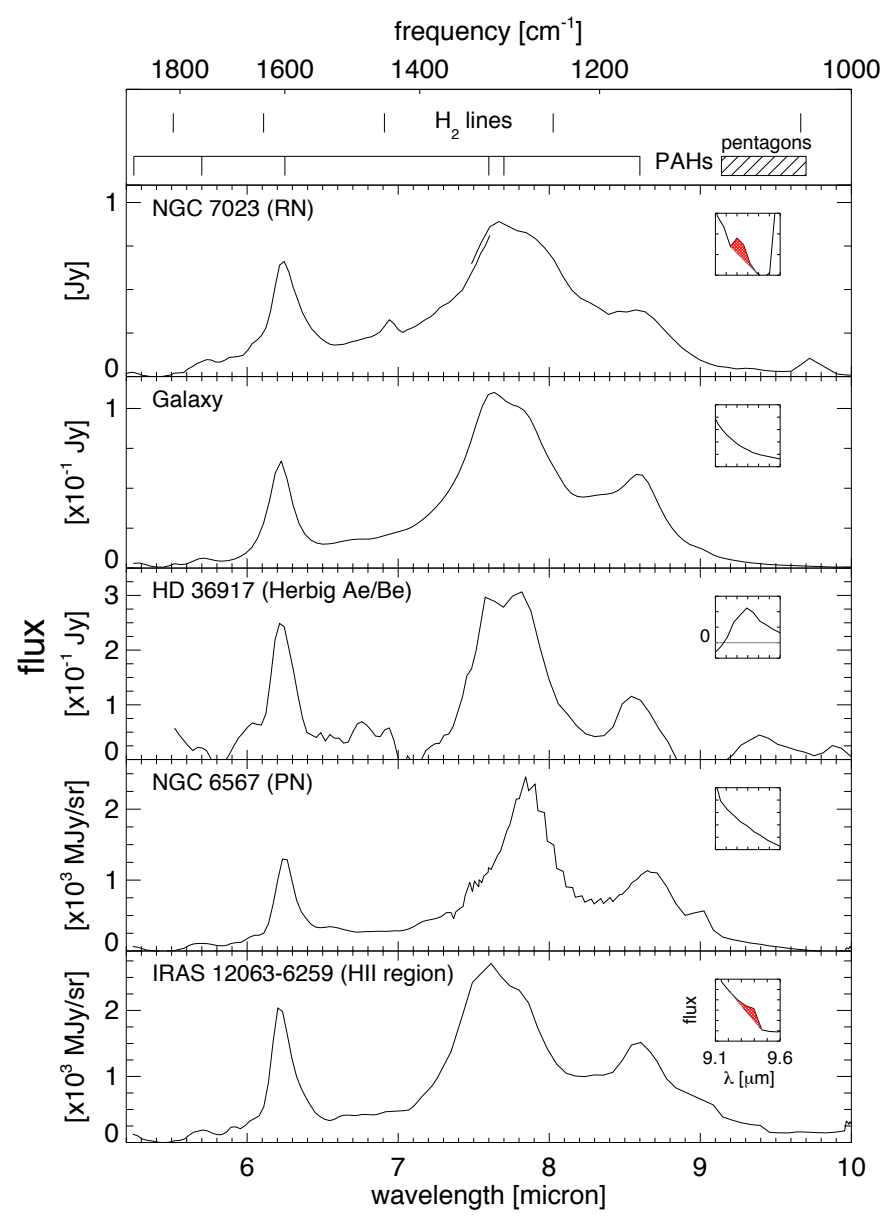

Fig. 6. 5.2-10 $\mu \mathrm{m}$ Spitzer-IRS low-resolution (SL) PAH emission spectra of a number of astronomical objects. Each panel indicates the name of the object and gives in parentheses the object-type $(\mathrm{RN}=$ reflection nebula; $\mathrm{PN}=$ planetary nebula). Top-most panel: positions of the major PAH bands and molecular hydrogen lines, with the hashed area indicating the region where possible emission from pentagon containing PAHs could be expected. The inset in each panel zooms in on this region and for NGC 7023 and IRAS 12063-6259 a tentative band is highlighted using a red double hash. See Sect. 4.4 for details.

to the existence of interstellar pentagon containing aromatic species. In addition, it could be argued that this also provides grounds for the existence of other defects in pristine hexagonal molecules, such as seven- or eight-membered rings (Ricca et al. 2011). Thus, the interstellar population of aromatic molecules containing pentagons is likely made up of those potentially generated in the ejecta of carbon-rich stars, which are modified and augmented in subsequent evolutionary phases of the star and planet forming process through erosion of large aromatic species. Hence, suggesting a link between astrophysical environment and the make up of the population of pentagon containing aromatic species. However, issues exist in marrying the observed bands to such species, which currently hampers their use as astrophysical probes.

Evidence for aromatic species being formed in the ejecta of carbon-rich stars has been mostly indirect. Although, some success is reported for observations on Tu Tau; a binary star system hosting a carbon-rich AGB star and a blue companion (Buss et al. 1991; Speck \& Barlow 1997; Boersma et al. 2006). Knowledge available from the combustion community has been 
used to explain the bottom-up formation of PAHs in the outflows of such stars (e.g., Frenklach \& Feigelson 1989; Cherchneff et al. 1992) and suggests that pentagon-containing PAH species are also produced (Bouwman et al. 2015; Johansson et al. 2018; Schulz et al. 2019; McCabe et al. 2020). Inclusion of pentagons can also be achieved through top-down erosion of large aromatic species, which is considered the prime mechanism for creating fullerenes (Berné \& Tielens 2012). Here, the suggested first step is the complete loss of hydrogen (Berné \& Tielens 2012).

The laboratory study performed here on $\mathrm{Rubi}^{\bullet+}$ and the study presented elsewhere on the diindenoperylene cation (Zhen et al. 2018) point to a band near $9.3 \mu \mathrm{m}$ that potentially could be used as a tracer for pentagon-containing aromatics. Scrutinizing PAHdb supports the presence of a distinct band near this wavelength in species like $\mathrm{Rubi}^{\bullet+}$ and diindenoperylene that have harbors. Our search for this feature in a limited sample of Spitzer-IRS spectra only shows a tentative detection of the feature in two sources, namely the H II-region IRAS 120636259 and the reflection nebula NGC 7023. Obviously, a single band does not justify identification and contributions from other PAHs, including nonpentagon and pentagon-containing dehydrogenated ones, to the emission observed around $9.3 \mu \mathrm{m}$ are not excluded as an exhaustive search and classification of such species is beyond the scope of this paper. Nonetheless, the band at $16.4 \mu \mathrm{m}$ that has also been linked to pentagons (Van Kerckhoven et al. 2000) could provide a handle on the connection of the $9.3 \mu \mathrm{m}$ band with interstellar pentagon-containing aromatics. Notably, the James Webb Space Telescope (JWST), which is set to launch in 2021, will with its superior sensitivity and spectral resolution provide new opportunities to search for and fully characterize the weak $9.3 \mu \mathrm{m}$ feature.

\section{Summary and conclusions}

We studied the IR multi-photon dissociation spectrum of Rubi ${ }^{\bullet+}$, which contains two pentagons fused to hexagons that are participating in the aromaticity of the molecule. It has been shown here, and elsewhere (Zhen et al. 2018), that pentagon-containing PAHs have IR activity between $1000-1200 \mathrm{~cm}^{-1}(\sim 9.3 \mu \mathrm{m})$. The DFT computed spectrum of Rubi ${ }^{\bullet+}$ and spectra available from the NASA Ames PAH IR Spectroscopic Database (PAHdb) associate the band with a so-called harbor mode. Here, two protruding hydrogen atoms are situated like breakwaters protecting a harbor bounded by either three (Type I) or four (Type II) walls; one of which belongs to a pentagon. The mode is dominated by in-plane vibrations of both the breakwater hydrogens and the carbon atoms associated with the pentagons.

Examining the low-resolution Spitzer-IRS spectra from a sample of five astronomical sources shows a tentative detection of the feature near $9.3 \mu \mathrm{m}$ in the spectrum of the reflection nebula NGC 7023 and that of the H II-region IRAS 120636259. After obtaining an estimate for the 9.3/6.2 $\mu \mathrm{m}$ PAH band strength ratio of $0.16 \pm 0.019$ for the harbor containing species in PAHdb we compute some $1-3 \%$ of the $6.2 \mu \mathrm{m}$ PAH band arising from such pentagon-containing aromatics. The strength of the $9.3 \mu \mathrm{m}$ feature is then $0.5 \%$ of the $6.2 \mu \mathrm{m}$ band at best. While the detected features are considerably feeble, PAHs with pentagons differently configured than Rubi $^{\bullet+}$ could still contribute in other ways to the interstellar spectra. Either way, pentagon containing aromatic species could prove to be a valuable new probe for astrophysical conditions in a number of environments; from their formation in the ejecta of carbon-rich stars and their subsequent modifications when moving through the different stages of the star- and planet-forming process to eventually end up being converted into fullerenes. The launch of JWST will provide new opportunities to search for and characterize interstellar pentagon features.

Acknowledgements. JB acknowledges the Netherlands Organisation for Scientific Research (Nederlandse Organisatie voor Wetenschappelijk Onderzoek, NWO) for a VIDI grant (grant number 723.016.006). CB is grateful for an appointment at NASA Ames Research Center through the San José State University Research Foundation (NNX17AJ88A) and acknowledges support from NASA's ADAP program (NNH16ZDA001N). AGGMT acknowledges support through the Spinoza premie of NWO. MB acknowledges the European Union (EU) and Horizon 2020 funding awarded under the Marie Skłodowska-Curie action to the EUROPAH consortium (grant number 722346). This work makes use of data and tools provided by the NASA Ames PAH IR Spectroscopic Database, which is supported through a directed Work Package at NASA Ames titled: "Laboratory Astrophysics - The NASA Ames PAH IR Spectroscopic Database". This work was sponsored by NWO Exact and Natural Sciences for the use of supercomputer facilities (grant number 16638 and 17676). The authors gratefully thank the staff at FELIX for their on-site support.

\section{References}

Allamandola, L. J., Tielens, A. G. G. M., \& Barker, J. R. 1985, ApJ, 290, L25

Allamandola, L. J., Tielens, A. G. G. M., \& Barker, J. R. 1989, ApJS, 71, 733

Andersson, M. P., \& Uvdal, P. 2005, J. Phys. Chem. A, 109, 2937

Bauschlicher, Jr., C. W., Ricca, A., Boersma, C., \& Allamandola, L. J. 2018, ApJS, 234, 32

Berné, O., \& Tielens, A. G. G. M. 2012, Proc. Nat. Acad. Sci., 109, 401

Boersma, C., Hony, S., \& Tielens, A. G. G. M. 2006, A\&A, 447, 213

Boersma, C., Bouwman, J., Lahuis, F., et al. 2008, A\&A, 484, 241

Boersma, C., Bauschlicher, C. W., Allamandola, L. J., et al. 2010, A\&A, 511, A32

Boersma, C., Bregman, J. D., \& Allamandola, L. J. 2013, ApJ, 769, 117

Boersma, C., Bauschlicher, Jr., C. W., Ricca, A., et al. 2014, ApJS, 211, 8

Bouwman, J., Bodi, A., Oomens, J., \& Hemberger, P. 2015, Phys. Chem. Chem. Phys., 17, 20508

Bouwman, J., de Haas, A. J., \& Oomens, J. 2016, Chem. Commun., 52, 2636

Bouwman, J., Castellanos, P., Bulak, M., et al. 2019, A\&A, 621, A80

Buss, Jr., R. H., Tielens, A. G. G. M., \& Snow, T. P. 1991, ApJ, 372, 281

Cami, J., Bernard-Salas, J., Peeters, E., \& Malek, S. E. 2010, Science, 329. 1180

Castellanos, P., Candian, A., Zhen, J., Linnartz, H., \& Tielens, A. G. G. M. 2018, A\&A, 616, A166

Cherchneff, I., Barker, J. R., \& Tielens, A. 1992, ApJ, 401, 269

Commodo, M., Kaiser, K., De Falco, G., et al. 2019, Comb. Flame, 205, 154

de Haas, A. J., Oomens, J., \& Bouwman, J. 2017, Phys. Chem. Chem. Phys., 19, 2974

Doroshenko, V. M., \& Cotter, R. J. 1996, Rapid Commun. Mass Spectrom., 10, 65

Ekern, S. P., Marshall, A. G., Szczepanski, J., \& Vala, M. 1998, J. Phys. Chem. A, 102,3498

Frenklach, M., \& Feigelson, E. D. 1989, ApJ, 341, 372

Frisch, M., Trucks, G., Schlegel, H. B., et al. 2009, Gaussian 09, Revision A. 02 (Wallingford, CT: Gaussian Inc.), 19, 227

Galliano, F., Madden, S. C., Tielens, A. G. G. M., Peeters, E., \& Jones, A. P. 2008, ApJ, 679, 310

Galué, H. A., Rice, C. A., Steill, J. D., \& Oomens, J. 2011, J. Chem. Phys., 134, 054310

Houck, J. R., Roellig, T. L., van Cleve, J., et al. 2004, ApJS, 154, 18

Hudgins, D. L., \& Allamandola, L. J. 1995a, J. Phys. Chem., 99, 3033

Hudgins, D. L., \& Allamandola, L. J. 1995b, J. Phys. Chem., 99, 8978

Hudgins, D. L., Sandford, S. A., \& Allamandola, L. J. 1994, J. Phys. Chem., 98, 4243

Hudgins, D. M., \& Allamandola, L. J. 1997, J. Phys. Chem. A, 101, 3472

Hudgins, D. M., Bauschlicher, C. W., Allamandola, L. J., \& Fetzer, J. C. 2000, J. Phys. Chem. A, 104, 3655 
Joblin, C., Boissel, P., Leger, A., D’Hendecourt, L., \& Defourneau, D. 1995, A\&A, 299, 835

Joblin, C., Tielens, A. G. G. M., Geballe, T. R., \& Wooden, D. H. 1996, ApJ, 460, L119

Johansson, K. O., Head-Gordon, M. P., Schrader, P. E., Wilson, K. R., \& Michelsen, H. A. 2018, Science, 361, 997

Juhász, A., Bouwman, J., Henning, T., et al. 2010, ApJ, 721, 431

Kaiser, R. I., Parker, D. S., \& Mebel, A. M. 2015, Annu. Rev. Phys. Chem., 66 , 43

Kennicutt, Jr., R. C., Armus, L., Bendo, G., et al. 2003, PASP, 115, 928

Leger, A., \& Puget, J. L. 1984, A\&A, 137, L5

Mackie, C. J., Chen, T., Candian, A., Lee, T. J., \& Tielens, A. G. G. M. 2018, J. Chem. Phys., 149, 134302

McCabe, M. N., Hemberger, P., Reusch, E., Bodi, A., \& Bouwman, J. 2020, J. Phys. Chem. Lett., 11, 2859

Moriarty, N. W., \& Frenklach, M. 2000, Proc. Combust. Inst., 28, 2563

Moutou, C., Verstraete, L., Léger, A., Sellgren, K., \& Schmidt, W. 2000, A\&A, 354, L17

Oepts, D., van der Meer, A. F. G., \& van Amersfoort, P. W. 1995, Infrared Phys. Technol., 36, 297

Oomens, J., van Roij, A. J. A., Meijer, G., \& von Helden, G. 2000, ApJ, 542, 404

Oomens, J., Meijer, G., \& von Helden, G. 2001, J. Phys. Chem. A, 105, 8302
Oomens, J., Tielens, A., Sartakov, B. G., von Helden, G., \& Meijer, G. 2003, ApJ, 591, 968

Oomens, J., Sartakov, B. G., Meijer, G., \& von Helden, G. 2006, Int. J. Mech. Sci., 254, 1

Parker, D. S. N., Kaiser, R. I., Troy, T. P., \& Ahmed, M. 2014, Angew. Chem. Int. Ed., 53, 7740

Ricca, A., Bauschlicher, Jr., C. W., \& Allamandola, L. J. 2011, ApJ, 729, 94

Richter, H., \& Howard, J. B. 2000, Prog. Energ. Comb. Sci., 26, 565

Schulz, F., Commodo, M., Kaiser, K., et al. 2019, Proc. Combust. Inst., 37, 885

Sellgren, K., Werner, M. W., Ingalls, J. G., et al. 2010, ApJ, 722, L54

Smith, J. D. T., Draine, B. T., Dale, D. A., et al. 2007, ApJ, 656, 770

Speck, A. K., \& Barlow, M. J. 1997, Ap\&SS, 251, 115

Tielens, A. G. G. M. 2008, ARA\&A, 46, 289

Van Kerckhoven, C., Hony, S., Peeters, E., et al. 2000, A\&A, 357, 1013

Werner, M. W., Uchida, K. I., Sellgren, K., et al. 2004, ApJS, 154, 309

Yang, T., Kaiser, R. I., Troy, T. P., et al. 2017, Angew. Chem. Int. Ed., 56, 4515

Zhen, J., Paardekooper, D. M., Candian, A., Linnartz, H., \& Tielens, A. G. G. M. 2014, Chem. Phys. Lett., 592, 211

Zhen, J., Castellanos, P., Bouwman, J., Linnartz, H., \& Tielens, A. G. G. M. 2017, ApJ, 836, 28

Zhen, J., Candian, A., Castellanos, P., et al. 2018, ApJ, 854, 27 


\section{Appendix A: Supporting information}

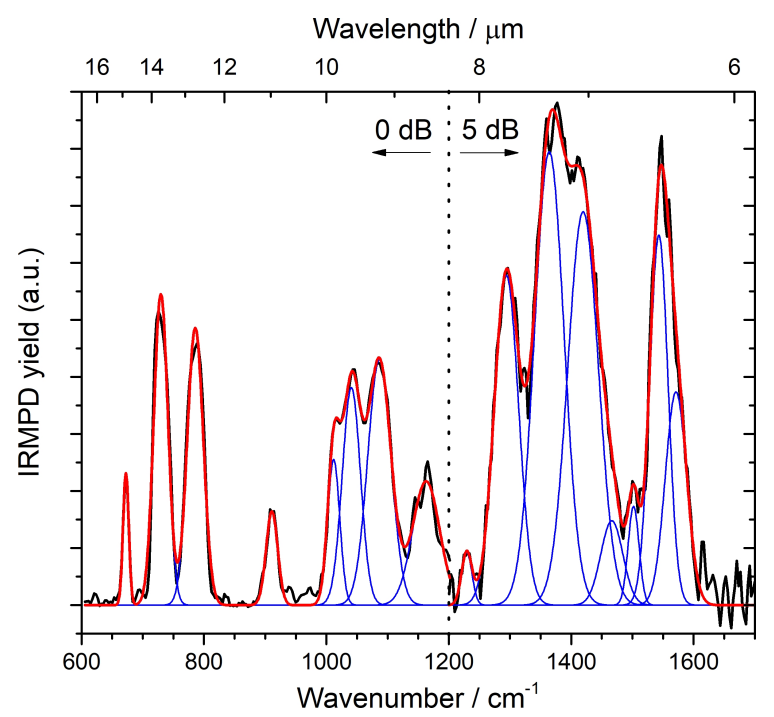

Fig. A.1. Spectrum of Rubi ${ }^{\bullet+}$ recorded over the $600-1700 \mathrm{~cm}^{-1}$ range shown together with a multi-component Gaussian fit to the measured data. The total fit is shown in red and the individual Gaussian line profiles are shown in blue.
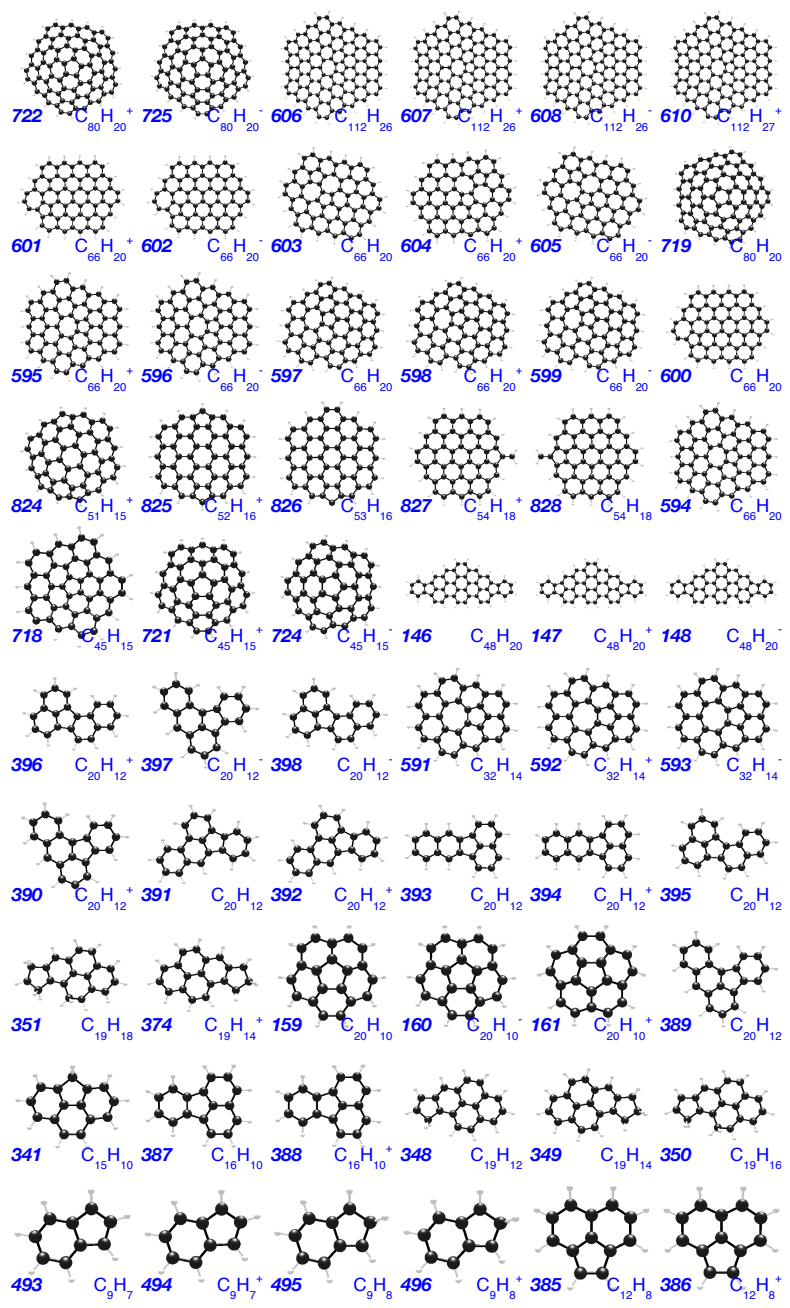

Fig. A.2. Chemical structures of the non-fully dehydrogenated pentagon containing species in PAHdb. The species unique identifier (UID) from PAHdb is shown in bold, together with its chemical formula. See Sect. 4.1 for details.

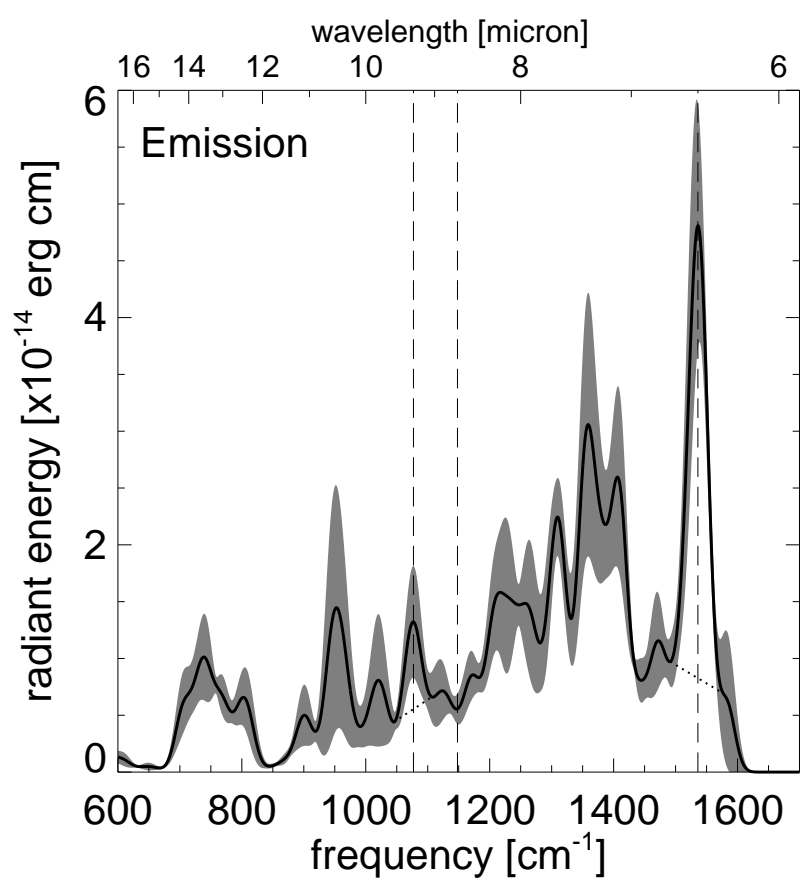

Fig. A.3. Summed $600-1700 \mathrm{~cm}^{-1}$ emission spectrum of the five pentagon containing cations in PAHdb with harbors (cf. Table 2). The bands have been convolved with a Gaussian line profile with a FWHM of $30 \mathrm{~cm}^{-1}$. The $1 \sigma$ variation around the mean is indicated by the gray shaded area. The 9.3, 8.6 and $6.2 \mu \mathrm{m}$ band positions have been indicated with the long-dashed lines. Straight-line continua used to isolate the 9.3 and $6.2 \mu \mathrm{m}$ PAH bands are shown as dotted lines. See Sect. 4.3 for details.

Figure A.1 shows a multi-component Gaussian fit to the experimental IRMPD spectrum. A good fit to the experimental data is obtained when including a total of 16 Gaussian components. Figure A. 2 presents the chemical structures of the 60 pentagon containing PAHs in version 3.00 of the library of computed spectra from PAHdb. The average spectrum of the five PAH cations with harbors is shown in Fig. A.3. 